\title{
SARS, SARS-Cov-2 and MERS are the Same Viral Species, Clades within Bat Beta-Coronaviruses
}

\author{
Michael J. Wise ${ }^{1,2 *}$ \\ ${ }^{1}$ Department of Computer Science \& Software Engineering, University of Western \\ Australia, Australia \\ ${ }^{2}$ Marshall Centre for Infectious Disease Research and Training, University of Western \\ Australia, Australia
}

\begin{abstract}
SARS-CoV-2 and SARS are human coronaviruses of zoonotic origin that first transferred from bats to humans in China. They are regarded as sister clades within the viral species Severe Acute Respiratory Syndrome-Related Coronavirus. A chain of bat beta-coronavirus strains links SARS and SARS-CoV-2. Here I report phylogenetic tree reconstructions in which SARS and SARS-CoV-2 are placed together with related bat strains. Single-species models are favoured over speciation models. This is also the case when the phylogenetic trees for MERS and its related beta-coronaviruses in camels, bats and hedgehogs is reconstructed, and even when the datasets are combined. Dated phylogenetic reconstructions suggest that both SARS-like and MERS-like beta-coronaviruses have been circulating for many years. I therefore propose that SARS, SARS-CoV-2 and MERS may be better thought of as sub-clades of bat beta-coronavirus that have on occasion transferred to humans. Looking specifically at SARS-CoV-2, there are also very high levels of conservation evident at the protein level, suggesting that multiple possible targets exist for vaccines or other therapeutics. Finally, these long-running reservoirs emphasise the need for ongoing wide-spread surveillance and reporting, and the importance of Planetary Health.
\end{abstract}

\section{Introduction}

Beta-coronaviruses SARS (Qin et al., 2003) and SARS-CoV-2 (Wu et al., 2020; Zhu et al., 2020) emerged suddenly in 2002 and 2019, respectively, and spread worldwide. They are now regarded as sister clades within the viral species Severe Acute Respiratory SyndromeRelated Coronavirus (Coronaviridae Study Group of the International Committee on Taxonomy of Viruses, 2020). It was first believed that SARS was transmitted from civets; civets are still regarded as the proximal host species, though it is now known that bats are the original host (Li et al., 2005). SARS-CoV-2 is also believed to have been transmitted from bats (Zhou et al., 2020).

Beta-coronaviruses are positive sense, single-strand RNA viruses, with a large (for RNA viruses) genomes encoding approximately 28 proteins. The proteins are encoded as a single polyprotein which includes the non-structural proteins, plus genes encoding a number of structural and accessory proteins The gene order is tightly constrained across the betacoronaviruses (Masters and Perlman, 2013), including SARS-CoV-2, though some genes, notably for accessory proteins orf8 and orf10, that are uniformly present in SARS-CoV-2 (Wu et al., 2020), are only variably present in SARS and related bat beta-coronaviruses. 
In coming to the finding that SARS and SARS-CoV-2 are sister clades the Coronaviridae Study Group of the International Committee on Taxonomy of Viruses used concatenations of three of the most conserved proteins: 3C-like protease 3CLpro (nsp5), nidovirus RdRp-associated nucleotidyltransferase NiRAN (nsp12), RNA-dependent RNA polymerase nsp12-RdRp (nsp12), superfamily 1 helicase with upstream Zn-binding domain nsp13ZBD and HEL1 (nsp13). In this study I instead have used concatenations of as many of the proteins as possible in a Bayesian framework, across both SARS and SARS-CoV-2, but also related bat beta-coronaviruses, to examine the question of whether a speciation event had occurred from the bat to the human beta-coronaviruses, and using tip dating based on dates of collection, to estimate when these events may have taken place. Similar analyses were also undertaken on concatenations of proteins from MERS strains and a set of related beta-coronaviruses, and then finally on concatenations involving the majority of proteins common to both sets of strains.

\section{Data and Methods}

\section{Data Used}

The proteome for the SARS-CoV-2 strain described in Wu et al. (2020) - corresponding to NCBI nucleotide entry NC_045512 - was obtained at https://viralzone.expasy . org/resources/Coronav/nCoV.fasta. The proteins, particularly the variable protein orf8, were used to search, via BLAST, for intact related SARS and bat beta-coronavirus proteins, and the corresponding closed genomes were retrieved. In addition, all the closed SARSCoV-2 genomes available at NCBI as at 9 March 2020 were obtained, which comprised 58 closed genomes spanning strains from patients in Australia, Brazil. China, Finland, India, Japan, Nepal, South Korea, Sweden, Taiwan and USA. The only other proviso was that there had to be date of collection, which included the month of collection, though for two strains the month was estimated as the month prior to date of submission of the sequence (which is plausible for the early SARS strains). The complete set contained 74 strains: 58 SARS-CoV-2, 2 SARS, 12 bat beta-coronaviruses and 2 related beta-coronaviruses found in civets. The complete list can be found as Table 1 in the Supplementary Data. A Python program, with Exonerate (Slater and Birney, 2005) as its underlying engine, was used to extract the translated protein sequences from each genome, based on the proteome corresponding to the genome NC_045512. The sets of proteins corresponding to the respective template proteins were aligned using Clustal Omega (Sievers et al., 2011), with the aligned proteins corresponding to each strain being concatenated. The final protein concatenations, 10,544aa, contained all the proteins except orf10 as it was absent from too many of the bat beta-coronavirus and SARS strains.

For the MERS dataset, proteins from strain HCoV-EMC/2012 (NC_019843) were used as the prototypes, resulting in a set of taxa from 14 MERS and related camel (seven), bat (fourteen) and hedgehog (three) beta-coronaviruses. The list of MERS-like strains can be found as Table 2 in the Supplementary Data, with the list of proteins used in the analyses listed in Supplementary Data Table 3. 


\section{Measuring Mean Protein Evolutionary Distance and Sequence Conser- vation in SARS-CoV-2}

The Mean Protein Evolutionary Distance (MeaPED) metric (Wise, 2013) can be used to measure the relative evolutionary pressure on proteins, i.e. the Darwinian pressure that exists on viral proteins to change due to action of the host immune system versus the countervailing requirements for proteins not to change so they can fulfil their functions. For the MeaPED analysis of SARS-CoV-2 alone, a larger set of genomes was obtained comprising 2,051 genomes available from NCBI as at 1 May, 2020. After removing poor quality genomes (defined as those with 10 or more contiguous $\mathrm{N}$ nucleotides), 1,412 genomes remained for MeaPED analysis after the protein translations had been extracted. MeaPED scores, Adjusted MeaPED scores (to account for duplicate protein sequences) and Adjusted MeaPED scores per 100aa, were computed for each of the proteins in the dataset. While the scores are relative, they can be compared to the values obtained in Wise (2013) using the same methods.

A second way of looking at sequence conservation is to count the number of contiguous, perfectly conserved sites, in multiple-sequence alignments of the different proteins. That is, the number of adjoining columns with identical amino acids down the respective columns were counted, with a minimum count of 3 adjoining perfect sites.

\section{Phylogenetic reconstructions}

While MeaPED can provide information about conservation at the protein level - which was here applied just to SARS-CoV-2 - an important question concerning the evolutionary biology of SARS-CoV-2, SARS and related bat beta-corona viruses is whether the former represent a new species. Also of interest is the dating of these events. Bayesian modelling was undertaken using BEAST 1.10.4 (Suchard et al., 2018). To set up the parameters of the model, the first step was to determine the per-site substitution model, for which I used modelgenerator v0.85 (Keane et al., 2006). The most likely substitution model turned out to be the JTT matrix with a gamma distribution used to model inter-site heterogeneity and a parameter for the percentage of invariant sites.

BEAST itself was then used to determine the most appropriate models for the clock and population based on the log-likelihood values. The following clock models where tested: strict clock, and uncorrelated relaxed clock with exponential, log-normal and gamma distributions (Drummond et al., 2006). The population models tested where: constant, exponential growth, expansion growth and Bayesian Skyline coalescent models (4 segments, representing the 4 viruses and hosts) and the Birth/Death and Yule speciation models. Initially, all combinations of clock and population models were tested with chain length $5,000.000$ to determined the most likely clock model.

Then, each of the population models was tested assuming the best clock found in the previous stage. In this case, each experiment was run 5 times with a chain length of $10,000,000$. Computations were judged not to have converged if the effective sample size (ESS) was less than or equal to 100, irrespective of burn-in, while log-likelihood value differences less than 5 were deemed to be indistinguishable. In a small number of cases, convergence did occur, but to a local minimum which was higher than average. Because these results would substantially skew mean values, they too were ignored. 
In the final stage, a dated analysis (based on tip-dating) was undertaken via repeated experiments with a chain length of 20,000,000. Logcombiner was used to pool the trees, after which TreeAnnotator was used to compute the final tree. BEAST logs were analysed using Tracer (Rambaut et al., 2018) and the trees were visualised using FigTree https: //github.com/rambaut/figtree/releases.

A similar analysis was undertaken for the MERS-related dataset, and finally for a dataset combining the SARS-like and MERS-like datasets, albeit for protein concatenations involving the 19 proteins the two sets have in common (listed in Table 3 in the Supplementary Data).

\section{Data and Application Availability}

The program used to compute MeaPED scores can be found at https://github.com/ mw263/MeaPED. The data used in the study can be found at https://cloudstor .aarnet. edu.au/plus/s/ZDi6EHLOqs0HILu for the duration of the review process. Once the paper has been accepted for publication and the final data storage requirements are known, the data will be transferred to the University of Western Australia Repository and a DOI will be minted and reported here.

\section{Results}

\section{Parameter Choices}

For the SARS-like dataset, the most likely clock model turned out to be an uncorrelated relaxed clock with a gamma distribution. (Complete results can be found in Supplementary Data Table 4). The uncorrelated relaxed clock with gamma distribution, combined with the other parameters, was used to test the different population models (averaged over 5 experiments with chain length 10,000,000 each). At this stage it was evident that single species coalescent models were favoured over both speciation models, but the Birth/Death model was none-the-less carried forward to the next stage for more extensive testing. As can be seen from Table 5 in the Supplementary Data, the simpler coalescent models (constant, exponential growth and expansion growth) were significantly more likely than the Birth/Death speciation model. The exponential growth coalescent model was chosen for the dated analysis as it includes the possibility of a constant (or near constant) population

The MERS-like data set achieved the remarkable result that all the population models were equally likely after rounding to the nearest integer log-likelihood value (Table 6 in the Supplementary Data). In this case, the exponential growth coalescent was also chosen for the dated analysis.

For the 112 concatenations in the combined SARS-like and MERS-like data set, the most likely model was the Bayesian Skyline coalescent model with 8 piecewise linear segments representing the 8 viruses and hosts in the combined dataset (Table 7 in the Supplementary Data). 


\section{Mean Protein Evolutionary Distance and Identical Subsequences}

The MeaPED analysis of the 1,412 SARS-CoV-2 genomes revealed a remarkable degree of conservation at the protein level (Table 1). Looking at the range of Adjusted MeaPED per 100aa (Wise, 2013) values, the most variable protein is orf8, suggesting that it is surface exposed. On the other hand, the Adjusted MeaPED per 100aa score for orf8, 0.00512, is very low and comparable with the most variable dengue virus proteins. By contrast, the surface exposed spike protein had an Adjusted MeaPED Score per 100 aa of 0.00001 , while the papain-like protease (plpro) also had a an Adjusted Mean Score per 100 aa of 0.00001. This is more conserved than even measles virus - also an enveloped single strand RNA virus (negative sense in this case) - or any single serotype of dengue virus, (an enveloped, positive sense, single strand RNA virus which has to survive in two hosts), and is comparable with the double-stranded DNA polyomavirus (see Supplementary data in (Wise, 2013)).

In addition to the MeaPED data, Table 1 also shows, for each protein, the length of the longest unbroken sequence of identical residues (i.e. the same residue for all taxa at a given site), and the total length of all such sequences with a minimum length of $3 \mathrm{aa}$. The results mirror the MeaPED values, with more perfectly conserved amino acids in the more conserved proteins, but a remarkable degree of conservation over all.

\section{Dated BEAST Phylogenetic Tree Construction}

A tip-dated phylogenetic tree construction based a chain length of 20,000,000 was undertaken multiple times on the SARS-like and MERS-like datasets. For each experiment burn-in was adjusted so that the ESS value was at least 100, and preferably over 200. A total of 21 successful SARS-like constructions yielded a combined ESS for the log-likelihood of the tree of 3,121. The TMRCA (the most recent common ancestor), i.e. root, shown in Figure 1, had an age corresponding to the year 1985 and had an ESS of 181 . The 95\% interval for the root was 1969 ... 1997.

For the MERS-like dataset, the root corresponded to the year 1837 with a $95 \%$ confidence range of 1622 ... 2005, based on 25 computed trees. The ESS for the root age was for 137, with ESS of the tree's log likelihood was 3,000. The tree is shown as Figure 2.

To reduced the extrapolation effect from a narrow range of tip dates, when constructing the dated phylogenetic tree for the combined dataset, shown in Supplementary Data Figure 3 , tip dating was retained, but in addition, gamma distributed calibration priors were placed on most recent common ancestor nodes for the two trees. In this case 30 trees were computed. The root of the combined tree had a root age corresponding to 1368 with a 95\% confidence interval of $894 \ldots$ 1819. The root had ESS of 109. Interestingly, the ages of the most recent common ancestors corresponding to the SARS-like and MERS-like clades where dragged back to 1961 and 1797, respectively.

The final experiment was to undertake a reconstruction of the Bayesian Skyline model for the population of the combined strains. The output is shown in Figure 3. 


\section{Discussion}

\section{Conservation Analyses}

Looking at the Mean Protein Evolution Data for the large SARS-CoV-2 dataset it is clear that there is a very high level of conservation across the proteins produced by this virus. More practically, the stretches of perfectly conserved residues suggests that there is no shortage of possible targets for drugs or vaccines, though of course efficacy and cross reactivity are significant issues.

\section{Phylogenetic Analyses}

The first, and most striking, finding from the Bayesian phylogenetic tree construction experiments is that for both the SARS-like and MERS-like datasets, and even for the combined dataset, there is less support for speciation events having occurred than there is for coalescence models involving a single species with parallel sister clades (or quasi-species). Furthermore, as is evident from Figure 3, the size of the total population of these viruses, predominantly in bats, has largely remained constant over the years, except for a sharp process of narrowing that began in 2009, accelerating in 2013 to reach a minimum in 2017. The spread of SARS-CoV-2 in humans has seen a rebound in the population.

We can see from the unrooted tree for the SARS-like dataset (shown in Supplementary Data Figure 1), that there is chain of bat beta-coronavirus strains linking SARS and SARS-CoV-2, with the civet strains close to SARS. No proximal animal strain has thus far been definitively identified for SARS-CoV-2. Given the initiation dates of SARS and SARSCoV-2 it is tempting to suggest that a circulating bat beta-coronavirus that transitioned to humans to become SARS in 2002 later evolved into the species that transitioned into humans, becoming SARS-CoV-2. However, from Figure 1 here it is evident that the bat beta-coronaviruses which became SARS and SARS-CoV-2 were circulating independently, having derived from a progenitor species (likely from bats) that had median origin date of 1985 (95\% interval: 1969 ... 1997). A similar picture emerges from the MERS-like strains phylogenetic tree (Figure 2, with unrooted tree as Figure 2 in the Supplementary Data), where the route to MERS is studded with bat beta-coronavirus strains, and includes an interesting diversion into hedgehogs.

\section{Conclusions}

From these analyses it is possible to reach a number of conclusions:

- Given that all the beta-coronaviruses listed here are more likely to constitute a single species than for a speciation event to have occurred, the primary beta-coronavirus linking all the others is from bats; the other SARS-like and MERS-like viruses resemble subclasses of the dominant bat beta-coronaviruses.

- SARS-CoV-2, and most likely the other bat beta-coronaviruses, are highly conserved at the protein level, suggesting multiple possible targets exist for vaccines or other therapeutics. This level of conservation also suggests that if an efficacious vaccine 
can be found, a concerted vaccination campaign, similar to that waged against polio, could see the elimination of SARS-CoV-2 (and potentially also MERS).

- The fact that the progenitors for these viruses have been circulating in bats for many years suggests that the transfer to humans is a rare event, having only notably occurred three times, albeit relatively recently. By the same token, the transfer can happen again, which emphasises the role of surveillance and communication of findings. From the data used in this study, SARS-like bat beta-coronavirus strains and MERS-like strains are both circulating in bats in China. It is entirely conceivable that they can also be found elsewhere, but no other data from systematic surveys has been published. It is therefore important that more such surveys be carried out and reported.

\section{Acknowledgements}

I am very grateful for the very open handed access I was granted to the new Nimbus facility at the Pawsey Supercomputer Facility, Perth, Western Australia.

\section{Data and Application Availability}

The program used to compute MeaPED scores can be found at https://github.com/ mw263/MeaPED. The data used in the study can be found at https://cloudstor aarnet. edu.au/plus/s/ZDi6EHLOqsOHILu. Once the paper has been accepted for publication and the final data storage requirements are known, the data will be transferred to the University of Western Australia Repository and a DOI will be minted and reported here.

\section{References}

Coronaviridae Study Group of the International Committee on Taxonomy of Viruses 2020. The species severe acute respiratory syndromerelated coronavirus: Classifying 2019ncov and naming it sars-cov-2. Nat Microbiol, 5: 536-544.

Drummond, A. J., Ho, S. Y. W., Phillips, M. J., and Rambaut, A. 2006. Relaxed phylogenetics and dating with confidence. PLoS Biol, 4(5): e88.

Keane, T. M., Creevey, C. J., Pentony, M. M., Naughton, T. J., and Mclnerney, J. O. 2006. Assessment of methods for amino acid matrix selection and their use on empirical data shows that ad hoc assumptions for choice of matrix are not justified. BMC Evol Biol, 6: 29.

Li, W., Shi, Z., Yu, M., Ren, W., Smith, C., Epstein, J. H., Wang, H., Crameri, G., Hu, Z., Zhang, H., Zhang, J., McEachern, J., Field, H., Daszak, P., Eaton, B. T., Zhang, S., and Wang, L.-F. 2005. Bats are natural reservoirs of sars-like coronaviruses. Science, 310: 676-679. 
Masters, P. S. and Perlman, S. 2013. Coronaviridae. In D. M. Knipe and P. M. Howley, editors, Fields Virology (6e), chapter 28, pages 825-858. Kluwer Health/Lippincott Williams \& Wilkins.

Qin, E., Zhu, Q., Yu, M., Fan, B., Chang, G., Si, B., Yang, B., Peng, W., Jiang, T., L1u, B., Deng, Y., Lu, H., Zhang, Y., Wang, C., Li, Y., Gan, Y., Li, X., Lo, F., Tan, G., Cao, W., Yang, R., Wang, J., Li, W., Xu, Z., Li, Y., Wu, Q., Lin, W., Chen, W., Tang, L., Deng, Y., Han, Y., Li, C., Lei, M., Li, G., Li, W., Lo, H., Shi, J., Tong, Z., Zhang, F., Li, S., Liu, B., Liu, S., Dong, W., Wang, J., Wong, G. K.-S., and Yu, J. 2003. A complete sequence and comparative analysis of a sars-associated virus (isolate bjol). Chinese Sci Bull, 48: 941-948.

Rambaut, A., Drummond, A. J., Xie, D., Baele, G., and Suchard, M. A. 2018. Posterior summarization in bayesian phylogenetics using tracer 1.7. Syst Biol, 67: 901-904.

Sievers, F., Wilm, A., Dineen, D., Gibson, T. J., Karplus, K., Li, W., Lopez, R., McWilliam, H., Remmert, M., Söding, J., and Thompson, J. D. 2011. Fast, scalable generation of high-quality protein multiple sequence alignments using clustal omega. Mol Syst Biol, 7: 539.

Slater, G. S. and Birney, E. 2005. Automated generation of heuristics for biological sequence comparison. BMC Bioinformatics, 6: 31 .

Suchard, M. A., Lemey, P., Baele, G., Ayres, D. L., Drummond, A. J., and Rambaut, A. 2018. Bayesian phylogenetic and phylodynamic data integration using beast 1.10. Virus Evol, 4(1): vey016.

Wise, M. J. 2013. Mean protein evolutionary distance: A method for comparative protein evolution and its application. PLoS One, 8: e61276.

Wu, F., Zhao, S., Yu, B., Chen, Y.-M., Wang, W., Song, Z.-G., Hu, Y., Tao, Z.-W., Tian, J.-H., Pei, Y.-Y., Yuan, M.-L., Zhang, Y.-L., Dai, F.-H., Liu, Y., Wang, Q.-M., Zheng, J.-J., Xu, L., Holmes, E. C., and Zhang, Y.-Z. 2020. A new coronavirus associated with human respiratory disease in china. Nature, 579: 265-269.

Zhou, P., Yang, X.-L., and Shi, Z.-L. 2020. A pneumonia outbreak associated with a new coronavirus of probable bat origin. Nature, 579: 270-273.

Zhu, N., Zhang, D., Wang, W., Li, X., Yang, B., Song, J., Zhao, X., Huang, B., Shi, W., and Lu, R. 2020. A novel coronavirus from patients with pneumonia in china, 2019. N Engl J Med, 382: 727-733. 
Table 1: Mean Protein Evolutionary Distance scores, MeaPED scores adjusted for duplicate proteins, and MeaPED adjusted scores per 100aa, together with data on the longest unbroken sequence of identical residues and the total number of identical residues in unbroken sequences of length 3 or more. Starting $\mathrm{N}=1,412$ taxa before removal of duplicates, leaving $\mathrm{N}$ taxa

\begin{tabular}{|cccccccc|}
\hline ID & Len & Mean & AdjMean & $\begin{array}{c}\text { AdjMean } \\
\text { per 100aa }\end{array}$ & $\begin{array}{c}\text { Longest } \\
\text { ident }\end{array}$ & $\begin{array}{c}\text { Total } \\
\text { ident }\end{array}$ \\
orf8 & 121 & 0.41637 & 0.0061925 & 0.00512 & 21 & 9 & 9 \\
orf14 & 73 & 0.039651 & 0.00073011 & 0.001 & 26 & 19 & 35 \\
orf6 & 61 & 0.036123 & 0.00025583 & 0.00042 & 10 & 16 & 47 \\
orf7a & 121 & 0.024293 & 0.00029248 & 0.00024 & 17 & 26 & 84 \\
orf9b & 97 & 0.018162 & 0.00016721 & 0.00017 & 13 & 27 & 83 \\
orf7b & 43 & 0.02371 & $6.72 \mathrm{E}-05$ & 0.00016 & 4 & 18 & 29 \\
nsp7 & 83 & 0.02028 & $8.62 \mathrm{E}-05$ & 0.0001 & 6 & 34 & 78 \\
orf3 & 275 & 0.0096301 & 0.00028645 & 0.0001 & 42 & 28 & 225 \\
nsp8 & 198 & 0.017727 & 0.00017576 & 0.00009 & 14 & 36 & 159 \\
nsp1 & 179 & 0.0061751 & 0.00014869 & 0.00008 & 34 & 32 & 122 \\
nsp9 & 113 & 0.015953 & $9.04 \mathrm{E}-05$ & 0.00008 & 8 & 36 & 105 \\
vemp & 75 & 0.020409 & $5.78 \mathrm{E}-05$ & 0.00008 & 4 & 35 & 72 \\
ncap & 419 & 0.0055722 & 0.00026835 & 0.00006 & 68 & 37 & 328 \\
nsp2 & 638 & 0.0052138 & 0.0002917 & 0.00005 & 79 & 40 & 548 \\
vme1 & 222 & 0.0088709 & 0.00010052 & 0.00005 & 16 & 79 & 206 \\
3cl & 306 & 0.0064698 & 0.00012372 & 0.00004 & 27 & 34 & 279 \\
nsp16 & 298 & 0.0066127 & 0.00012176 & 0.00004 & 26 & 31 & 268 \\
nsp6 & 290 & 0.0063886 & 0.00010406 & 0.00004 & 23 & 39 & 264 \\
hel & 601 & 0.0046206 & 0.00015053 & 0.00003 & 46 & 39 & 542 \\
nsp15 & 346 & 0.0050988 & $8.67 \mathrm{E}-05$ & 0.00003 & 24 & 34 & 323 \\
nsp14 & 527 & 0.0036444 & 0.00010324 & 0.00002 & 40 & 48 & 461 \\
nsp4 & 500 & 0.0039971 & $9.62 \mathrm{E}-05$ & 0.00002 & 34 & 54 & 458 \\
nsp10 & 139 & 0.0096791 & $2.06 \mathrm{E}-05$ & 0.00001 & 3 & 71 & 137 \\
plpro & 1945 & 0.0011788 & 0.00012189 & 0.00001 & 146 & 72 & 1773 \\
rdrp & 931 & 0.0025135 & $8.19 \mathrm{E}-05$ & 0.00001 & 46 & 81 & 877 \\
spike & 1273 & 0.001904 & 0.0001308 & 0.00001 & 97 & 125 & 1105 \\
\hline & & & & & & &
\end{tabular}




\section{Figures}

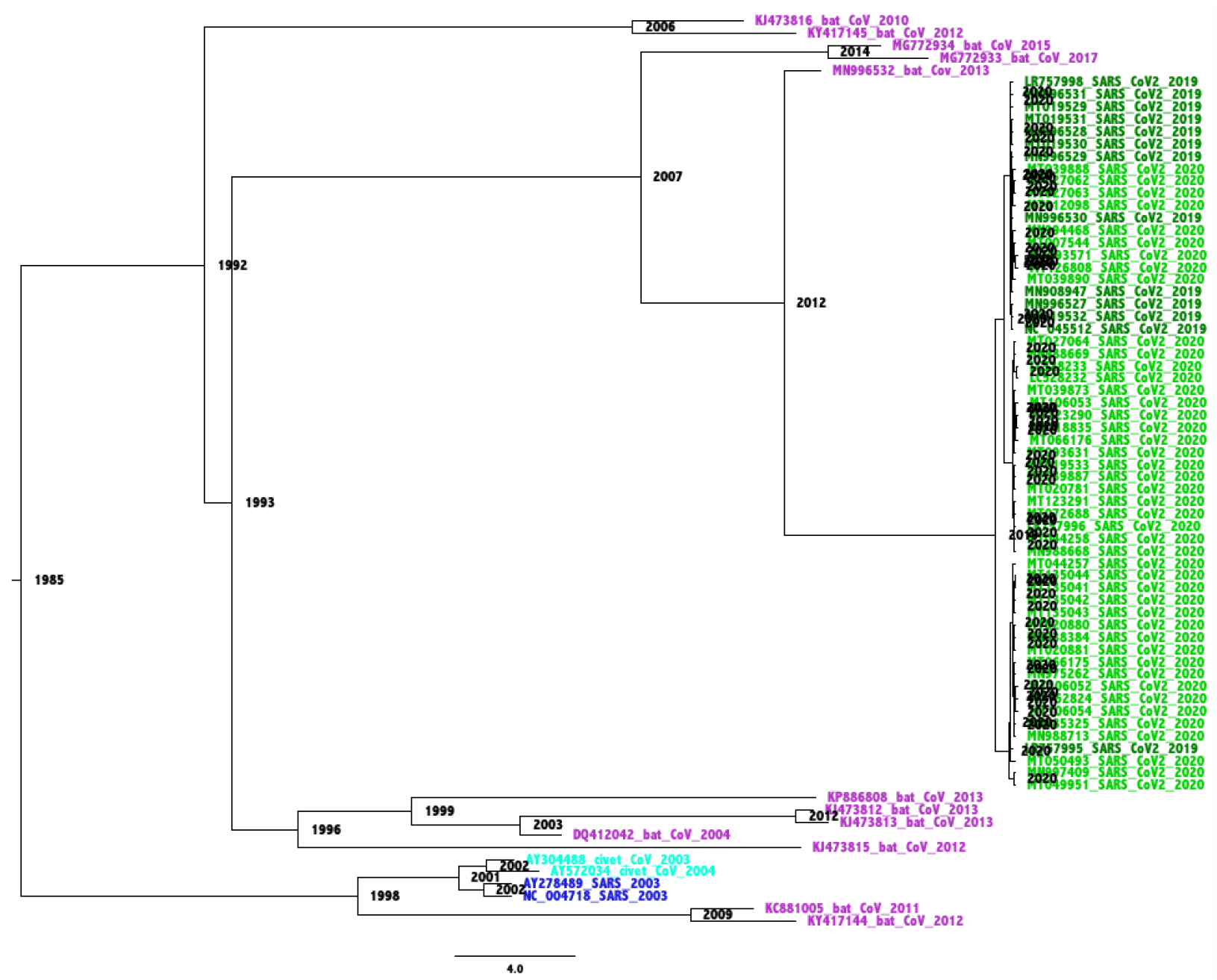

Figure 1: Phylogenetic Tree of SARS-like strains

A tip-dated phylogenetic tree of the 74 SARS-like created using BEAST 1.10.4. Median node ages are shown. SARS strains are shown in dark blue, related beta coronavirus strains from civet are shown in cyan, with strains from bats shown in mauve. SARS-CoV2 strains from 2019 are shown in dark green, while SARS-CoV-2 strains from 2020 are shown in light green 


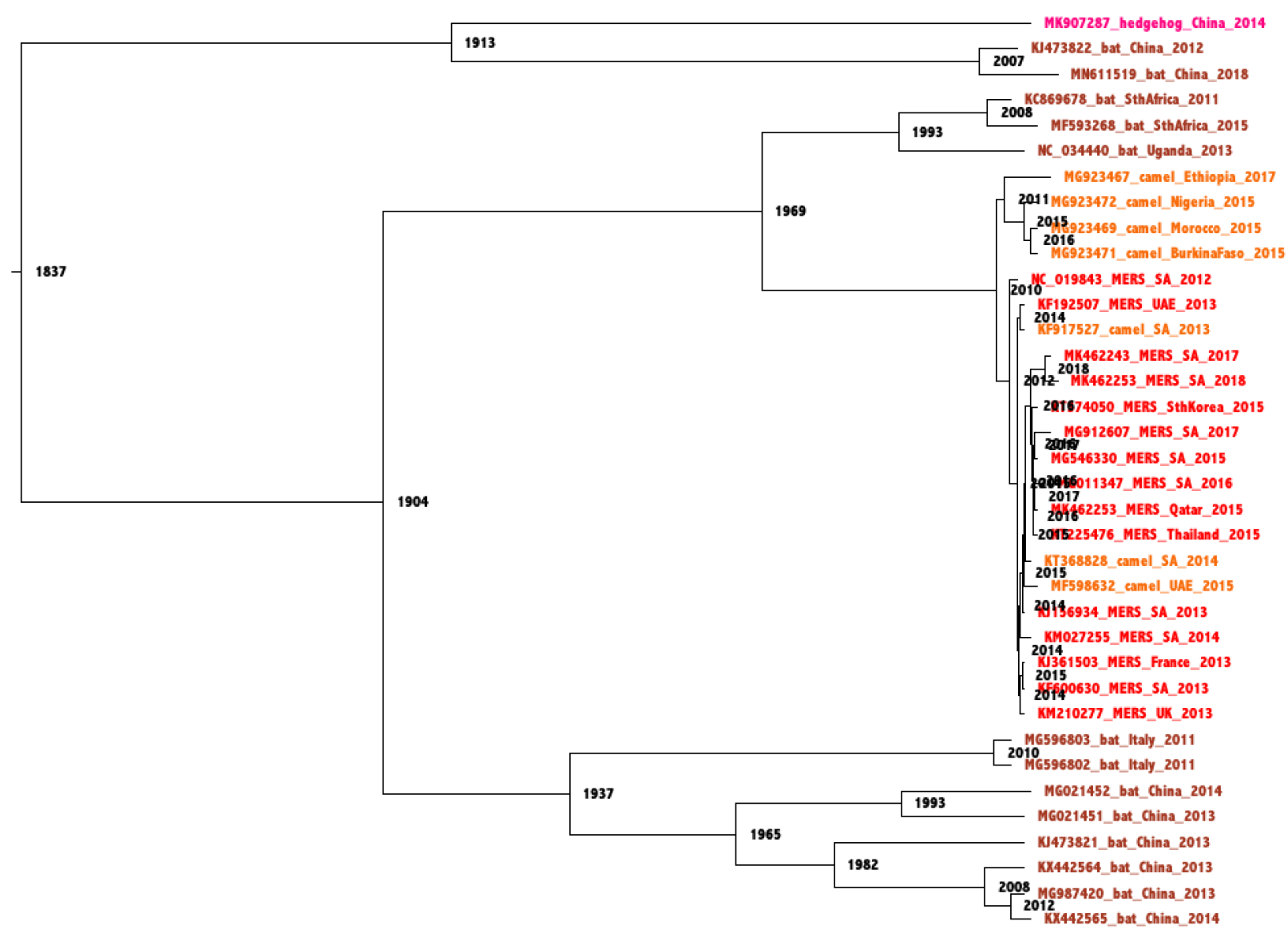

Figure 2: Phylogenetic Tree of MERS-like strains

A tip-dated phylogenetic tree of the 36 MERS-like created using BEAST 1.10.4. Median node ages are shown. MERS strains are shown in red, related beta coronavirus strains from camel are shown in orange, with strains from bats shown in brown and from hedgehogs in pink. 


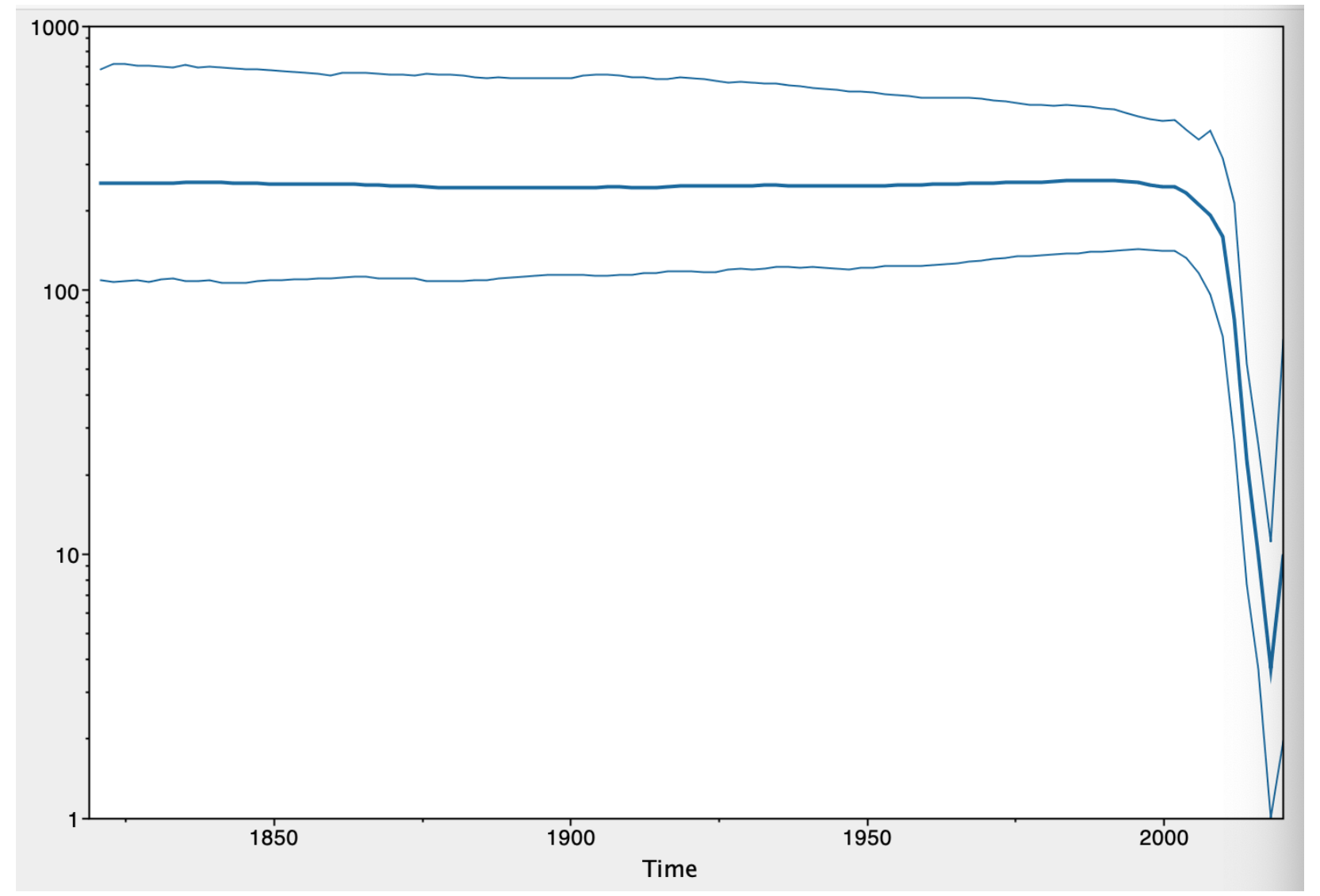

Figure 4: Population Profile of the Combined SARS-like and MERS-like strains The population size profile over time of the combined SARS-like and MERS-like beta coronaviruses $(\mathrm{N}=112)$ as computed by Trace 1.7.1. The dark blue line in the median value, while the lower and upper light blue lines are the 5\% and 95\% values. 